\title{
Desafios enfrentados por cuidadores de idosos: uma revisão integrativa
}

\author{
Iasmim Sofia Terceiro Nunes ${ }^{1 *} \bullet$, Gilmárcia Pinheiro Pereira ${ }^{2} \bullet$ \\ ${ }^{1}$ Enfermeira pela Universidade CEUMA, São Luís, Maranhão, Brasil. ${ }^{2}$ Enfermeira, Especialista em Saúde da Família, São Luís, Maranhão, \\ Brasil. *Autor para correspondência. E-mail: iasmimterceiro20@gmail.com
}

\begin{abstract}
Resumo: Introdução: Os cuidadores são componentes essenciais para a área da saúde, principalmente nas situações crônicas. O presente estudo teve como objetivo reconhecer os elementos causadores de sobrecarga no cuidador do idoso, avaliando os artigos dos anos de 2015-2020. Revisão: Em termos metodológicos, foi feita uma revisão integrativa realizada através de levantamento bibliográfico que se deu junto à Biblioteca Virtual de Saúde (BVS) acessando as bases de dados LILACS e portal e Scielo, e selecionando-se 10 artigos. Discussão: Entre os resultados obtiveram-se as evidências de que relação paciente-cuidador deve se pautar em planejamento que considere a saúde do cuidado, e que a sobrecarga pode ser evitada quando em melhor compensação dos serviços prestados e revezamento com outros cuidadores. Considerações finais: Concluiu-se que as causas de sobrecarga em cuidadores, predominantemente, são a do contexto de maior grau de dependência dos idosos, seguido de outros fatores como o perfil socioeconômico e a própria qualidade da saúde dos cuidadores.
\end{abstract}

Palavras-chave: cuidadores, idoso, sobrecarga.

\begin{abstract}
Introduction: Caregivers are essential components for the health area, especially in chronic situations. The present study aimed to recognize the elements that cause overload in the elderly caregiver, evaluating the articles from the years 2015-2020. Review: In methodological terms, an integrative review was carried out through a bibliographic survey that took place at the Virtual Health Library (VHL), accessing the LILACS and portal and SciELO databases, and selecting 10 articles. Discussion: Among the results, there was evidence that the patient-caregiver relationship should be based on planning that considers the health of care, and that overload can be avoided when better compensation for the services provided and relay with other caregivers. Final considerations: It was concluded that the causes of burden on caregivers, predominantly, are that of the context of a greater degree of dependence on the elderly, followed by other factors such as the socioeconomic profile and the quality of health of caregiver.
\end{abstract}

Keywords: caregivers, elderly, overload.

\section{Introdução}

Uma vida extensa em anos vem acompanhada de muitas possibilidades e desafios, principalmente na fase idosa. Quando há prolongamento dessas possibilidades, enfrentando-se o processo de envelhecimento, quase sempre a saúde do indivíduo se torna mais debilitada. Existe, contudo, alguns indícios que demonstram que os indivíduos idosos da atualidade vivenciam sua idade mais avançada mais satisfatoriamente que seus antepassados. Mesmo que os índices de inaptidão tenham reduzido nos países com elevado desenvolvimento econômico nos 30 anos anteriores, não ocorreu alteração considerável na inaptidão leve e moderada ao longo da mesma época (OPAS, 2018).

Segundo dados da Organização Mundial de Saúde - OMS, no período de 1950 a 2025, o número de idosos no país terá crescido em quinze vezes, ao passo que a população inteira em cinco. Deste modo, o país atingirá o sexto lugar no que se refere ao grupo de idosos, atingindo, em 2025, aproximadamente 32 milhões de indivíduos com 60 anos ou mais (Brasil, 2010).

De acordo com Gonçalves et al. (2013) quando nos referimos a idosos mais idosos, o grupo composto por esses indivíduos com 75 anos ou mais apontou um crescimento superior nos últimos dez anos, quando equiparado a totalidade dos indivíduos idosos. O grupo populacional composto por pessoas que chegaram à quarta idade (octogenários) tem comprovado hoje em dia que estão envelhecendo com mais autossuficiência para as atividades diárias, mesmo que em efêmera constância da condição de saúde.

Perante o exposto, principalmente no que se refere ao crescimento significativo dos idosos brasileiros, constatou-se a relevância de elaborar premissas para que o processo de envelhecimento aconteça com 
qualidade. Por isso, em 12 de julho de 2017, foi sancionada a Lei 13.466 que modifica o Estatuto do Idoso e determina preferência exclusiva para indivíduos maiores de 80 anos. Conforme a modificação, os maiores de 80 anos permanentemente terão suas necessidades respondidas com prioridade com relação aos outros idosos (Barbosa et al., 2017a).

A convivência doméstica e as atenções na quarta idade impactam a funcionalidade da situação familiar, o que leva a refletir em assimilações de qualidade regular ou intermediária de qualidade e estilo de vida dos cuidadores, e também dos próprios idosos nos diversos âmbitos e particularidades (Gonçalveset al., 2013).

O crescimento da perspectiva de vida repercute nas situações de vitalidade, morbidade e restrições funcionais nos idosos, aumentando a ocorrência de moléstias e incapacidades, com prováveis mudanças em vários setores de sua vida, precisando assim de dedicação contínua (Lana \& Schneider, 2014).

Segundo Loureiro et al. (2014), é conveniente enfatizar que o idoso dependente, seja de natureza física ou cognitiva de forma isolada, ou junção da dependência física e cognitiva, demandam grande empenho dos sistemas de assistência social, sendo eles aqui considerados sistemas de cuidado, para auxiliar nas dificuldades peculiares desse grupo.

A função de cuidador constitui a Classificação Brasileira de Ocupações - CBO segundo o código 5162. Numa concepção, mais abrangente da responsabilidade, a função do cuidador excede a elementar assistência das ações diárias dos pacientes, estejam eles nas residências ou em entidades onde precise de cuidado ou dedicação diária. A incumbência do cuidador é conduzir e ajudar o paciente a se cuidar, realizando pelo paciente apenas as ações que ele não seja capaz de realizar sozinho (Brasil, 2008).

De acordo com Yamashita et al. (2014), durante o acompanhamento ao idoso, diversos cuidadores informais enfrentam limitações em suas vidas particulares, ao responsabilizar-se de assistir e cumprir obrigações de maneira contínua sendo capaz de encarar circunstâncias de esgotamento, o que gera distanciamento de relações humanas, entrave na rede social, de convivência e entretenimento e gerar a sobrecarga.

O estado de sobrecarga se correlaciona ao nível de dependência do indivíduo, à medida que esteja mais dependente e afetado cognitivamente, maior será a exigência de auxílio dispensado ao paciente. Desse modo, intensifica a exigência de esforços, encurtando o momento que o cuidador tem para si próprio, elevando sua apreensão e a sobrecarga no tocante à atribuição de tutelar (Brandãoet al., 2017).

A observação de que os cuidadores são elementos importantes para o âmbito da saúde, especialmente nas circunstâncias de idosos com doenças crônicas e de tempo indeterminado, tem estimulado o estudo das dificuldades encaradas por eles. Esse entendimento seria capaz de auxiliar profissionais de saúde no intuito de adaptar o assessoramento concedido às precisões dos cuidadores, contribuindo para a melhoria de suas condições de vida. Diante disso, manifestou-se o interesse de estudar e aprofundar o conhecimento perante os desafios enfrentados por esses profissionais.

Com esse estudo se presume colaborar com uma avaliação crítica sobre a matéria em questão, proporcionando à comunidade acadêmica e aos profissionais da saúde uma perspectiva que pondere entre a qualidade do cuidado ao idoso e a prevenção de problemas por excesso de dependência e outros fatores. Nesse sentido, a presente revisão integrativa tem como objetivo identificar os elementos causadores de sobrecarga no cuidador do idoso.

\section{Revisão}

Trata-se de uma revisão integrativa da literatura. Este tipo de estudo tem sido considerado o mais abrangente entre as abordagens metodológicas disponíveis, uma vez que integra variados pontos de vistas, concepções, demonstração de evidências oriundas de investigações de um mesmo tema ou perspectiva de problema (Botelho et al., 2011).

Em outra abordagem, se diz ser caracterizada por um tipo de revisão da literatura que abrange descobertas de estudos realizados através de variadas metodologias, possibilitando aos revisores sumarizar resultados sem prejudicar a filiação epistemológica dos estudos mencionados (Soares et al., 2014).

Visando a identificação dos artigos de convergência com os desafios enfrentados por cuidadores de idosos foram consultadas as bases de dados virtuais presentes no Portal de Pesquisa da Biblioteca de Saúde (BVS-BIREME): Literatura Latino-Americana e do Caribe em Ciências da Saúde (LILACS) e Scientific Electronic Library Online (Scielo), no período de fevereiro a maio de 2020, mediante os seguintes descritores (DeCS): cuidadores, idoso e sobrecarga.

Foram incluídos artigos que abrangessem o tema, no período de 2015 a 2020, disponíveis na integra, no idioma português, abordando a temática e os objetivos propostos. Assim, a partir da amostra de 40 artigos, 
foram excluídos 30 estudos que mostraram dualidade entre duas ou mais bases de dados, tampouco se encaixaram no período de publicação e nem apresentavam afinidade com o propósito estabelecido por esta revisão. Portanto, foram selecionados10 artigos: 3 publicações oriundas da base Scielo e 7 da base LILACS.

Logo após, se empreendeu a discussão, ressaltando os aspectos comuns e as respectivas peculiaridades. Todo o caminho percorrido, por sua vez, se pautou por questões éticas, por se tratar de uma revisão integrativa de literatura que teve como preocupação registrar os dados essenciais referenciando os autores.

\section{Discussão}

Após análise, os artigos foram organizados em tabela, com o objetivo de sumarizar e confrontar os dados alcançados que equivaliam à temática orientadora do estudo. Inicialmente se fez uma categorização por tipo de estudo, com a indicação dos respectivos títulos, conforme a tabela 1:

Tabela 1. Distribuição de artigos selecionados segundo a base de dados, autores, tipo de estudo e título.

\begin{tabular}{|c|c|c|c|c|}
\hline Artigo & Base & Autores & Tipo de Estudo & Título \\
\hline (1) & LILACS & $\begin{array}{l}\text { Lino et al., } \\
\quad 2016\end{array}$ & Transversal. & $\begin{array}{l}\text { Prevalência de sobrecarga e respectivos fatores associados em } \\
\text { cuidadores de idosos dependentes, em uma região pobre do Rio } \\
\text { de Janeiro, Brasil. }\end{array}$ \\
\hline (2) & SCIELO & $\begin{array}{l}\text { Leite et al., } \\
\quad 2017\end{array}$ & Descritivo Transversal. & $\begin{array}{c}\text { A vulnerabilidade dos cuidadores de idosos com demência: } \\
\text { estudo descritivo transversal }\end{array}$ \\
\hline (3) & LILACS & $\begin{array}{l}\text { Lopes et al., } \\
\quad 2020\end{array}$ & $\begin{array}{l}\text { Observacional } \\
\text { quantitativo }\end{array}$ & $\begin{array}{l}\text { Associação entre a ocorrência de dor e sobrecarga em cuidadores } \\
\text { principais e o nível de independência de idosos nas atividades de } \\
\text { vida diária: estudo transversal. }\end{array}$ \\
\hline (4) & SCIELO & $\begin{array}{l}\text { Mendes et al., } \\
2019\end{array}$ & Analítico transversal. & $\begin{array}{l}\text { Sobrecargas física, emocional e social dos cuidadores informais } \\
\text { de idosos. }\end{array}$ \\
\hline (5) & LILACS & $\begin{array}{l}\text { Muniz et al., } \\
2016\end{array}$ & $\begin{array}{l}\text { Quantitativo do tipo } \\
\text { descritivo. }\end{array}$ & $\begin{array}{c}\text { Grau de sobrecarga dos cuidadores de idosos atendidos em } \\
\text { domicílio pela Estratégia Saúde da Família. }\end{array}$ \\
\hline (6) & LILACS & $\begin{array}{l}\text { Nunes et al., } \\
\quad 2019\end{array}$ & $\begin{array}{l}\text { Estudo qualitativo, } \\
\text { exploratório e descritivo. }\end{array}$ & $\begin{array}{c}\text { Fatores determinantes na transição situacional de familiares } \\
\text { cuidadores de idosos com doença de Parkison. }\end{array}$ \\
\hline (7) & LILACS & $\begin{array}{l}\text { Soares et al., } \\
\quad 2016\end{array}$ & $\begin{array}{l}\text { Analítico de corte } \\
\text { transversal. }\end{array}$ & $\begin{array}{l}\text { Cuidando do paciente com Síndrome Demencial: a sobrecarga do } \\
\text { cuidador principal. }\end{array}$ \\
\hline (8) & LILACS & $\begin{array}{l}\text { Santos et al., } \\
2019\end{array}$ & $\begin{array}{l}\text { Observacional, descritivo, } \\
\text { qualitativo. }\end{array}$ & $\begin{array}{c}\text { Sobrecarga de cuidadores idosos que cuidam de idosos } \\
\text { dependentes. }\end{array}$ \\
\hline (9) & LILACS & $\begin{array}{l}\text { Diniz et al., } \\
2018\end{array}$ & $\begin{array}{l}\text { Observacional, } \\
\text { comparativo, quantitativo. }\end{array}$ & $\begin{array}{l}\text { Estudo comparativo entre cuidadores formais e informais de } \\
\text { idosos. }\end{array}$ \\
\hline (10) & SCIELO & $\begin{array}{l}\text { Barbosa et al., } \\
\text { 2017b }\end{array}$ & $\begin{array}{l}\text { Descritivo, abordagem } \\
\text { quantitativa. }\end{array}$ & $\begin{array}{l}\text { Qualidade de vida relacionada à saúde dos cuidadores formais de } \\
\text { idosos institucionalizados em Natal, Rio Grande do Norte. }\end{array}$ \\
\hline
\end{tabular}

Fonte: elaborada pelos autores.

Em relação aos sujeitos da pesquisa, a maioria era composta exclusivamente por cuidadores, enquanto os demais eram constituídos tanto dos cuidadores quanto de idosos dependentes; e em todos os artigos se identificaram os elementos causadores de sobrecarga, sobretudo, foram destacadas alternativas de superação através de suporte social, revezamento e orientações eventuais de enfermeiros da Estratégia de Saúde da Família. No entanto, uma quantidade menor fez comparativo de sobrecarga entre cuidadores formais e informais, e relação da sobrecarga com o perfil sociodemográfico do cuidador.

Na tabela 2, destacam-se as variáveis causadoras de sobrecarga.

Dois estudos demonstraram a relação das características sociodemográficas com a sobrecarga de cuidadores (Mendes et al., 2019; Soares et al., 2016). Contudo, a sobrecarga advém de muitos fatores, como o pouco domínio sobre os problemas de saúde, em consequência da informalidade dos cuidadores, o longo período de cuidados despendidos, e a elevada dependência do idoso (Linoet al., 2016).

Além disso, quando o cuidador também é um idoso, tal condição se torna um fator potencial de sobrecarga (Santos et al., 2019). Embora, na maioria dos casos, as dores suportadas pelos cuidadores não sejam originadas do esforço com os cuidados ao idoso, tais distúrbios interferem na qualidade dessas práticas (Munizet al., 2016).

Essas breves noções de elementos causadores de sobrecarga foram evidenciadas por meio da exposição dos seguintes enfoques: 1) Perfil sociodemográfico, econômico e clínico do cuidador através de adoção de avaliação de sobrecarga; 2) A prevalência maior de sobrecarga nos cuidados com idosos com algum tipo de demência; 3) Comparativo de sobrecarga entre cuidadores formais e informais, e o cuidador sob a orientação da equipe de Estratégia da Família. 
Tabela 2. Distribuição dos artigos por elementos causadores de sobrecarga.

\begin{tabular}{|c|c|}
\hline Artigo & $\begin{array}{l}\text { Elementos causadores de sobregarga } \\
\end{array}$ \\
\hline (1) & $\begin{array}{l}\text { Ausência de colaboração de outros familiares, suporte social insuficiente; Sobrecarga maior quando se } \\
\text { cuidam de idosos que sofrem de decadência de cognição e grau elevado de depressão. }\end{array}$ \\
\hline$(2)$ & $\begin{array}{c}\text { Os cuidados aos idosos com demência, portadores de degradação cognitiva se colocam como os casos } \\
\text { prevalentes de sobrecarga em idosos. }\end{array}$ \\
\hline (3) & Quanto maior a dependência do idoso e o cuidador apresenta desconhecimento sobre a patologia \\
\hline (4) & $\begin{array}{l}\text { Aspectos sócio demográficos, econômicos e clínicos do cuidador influem na sobrecarga. Quando há vínculo } \\
\text { conjugal entre o cuidador e o idoso sob os cuidados deste. O nível de complexidade de eventual patologia do } \\
\text { idoso e a colaboração precária ou inexistente de terceiros, contribuem para uma sobrecarga. }\end{array}$ \\
\hline$(5)$ & $\begin{array}{c}\text { O tipo de doença que é portado pelo idoso sob os cuidados; a sobrecarga é proporcional a maior incapacidade } \\
\text { do doente. }\end{array}$ \\
\hline (6) & $\begin{array}{l}\text { Residir no mesmo domicílio do idoso em cuidados; maior dependência do idoso; famílias disfuncionais; } \\
\text { condições (estruturais socioeconômicas) inadequadas aos cuidados. }\end{array}$ \\
\hline (7) & $\begin{array}{l}\text { Os maiores níveis de sobrecarga estão em cuidadores de idosos com Síndrome Demencial e doença } \\
\text { pulmonar, associado com o grau de parentesco com o cuidador. }\end{array}$ \\
\hline$(8)$ & $\begin{array}{l}\text { Idade elevada do próprio cuidador (cuidador idoso); coabitação entre cuidador e idoso; e idoso com quadro } \\
\text { demencial, que denota maior grau de dependência. }\end{array}$ \\
\hline (9) & $\begin{array}{c}\text { A sobrecarga em cuidadores informais se deve ao fato de que não dispõe de melhor planejamento. Já a } \\
\text { sobrecarga dos cuidadores formais se deve ao fato de que a quantidade de idosos sob a sua responsabilidade } \\
\text { é maior. }\end{array}$ \\
\hline (10) & $\begin{array}{l}\text { Em instituições de longa permanência, os elementos de sobrecarga são a extensa jornada de trabalho e maior } \\
\text { número de idosos para cuidar. }\end{array}$ \\
\hline
\end{tabular}

Fonte: elaborada pelos autores.

\section{Enfoque 1- O perfil sociodemográfico, econômico e clínico do cuidador por meio da avaliação de sobrecarga}

Para averiguação da correspondência dos aspectos sociodemográficos, econômicos e clínicos com a sobrecarga em cuidadores de idosos, a literatura científica tem feito indicação do emprego de instrumentos destinados à obtenção de dados, a exemplo do Questionário de Avaliação da Sobrecarga do Cuidador Informal (QASCI). Quanto a esse modelo de perguntas, convém destacar que sua aplicação original se destinou aos cuidadores informais de familiares idosos, porém admite adaptações.

Elaborado em Portugal, mas possuindo validação e adaptação à realidade brasileira, a primeira parte da avaliação se configura no registro de informações relativas às idades dos cuidadores e dos idosos assistidos; as situações civis, identidade sexual, nível de instrução escolar, vínculos de parentesco, condição de moradia e compartilhamento de mesma residência entre cuidador e idoso; administração medicamentosa, tempo de recreação, religiosidade e renda (Soares et al., 2016).

Como estrutura principal, o questionário se compõe das seguintes perspectivas de abordagem: (a) impacto nas emoções dos cuidadores; (b) efeitos na vida particular dos cuidadores; (c) consequências financeiras para os cuidadores; (d) respostas e atitudes perante as demandas dos cuidados; (e) autoavaliação do desempenho das tarefas;(f) suporte de familiares; e (g) valoração própria do papel de cuidador. Observou-se que os questionamentos trabalham com indicadores de frequência, para registro de pontuação, em termos de "não/nunca", "raramente", “às vezes", "quase sempre" e "sempre". A literatura denomina tais perspectivas de abordagem como domínios de avaliação de sobrecarga (Monteiro et al., 2015).

Em relação ao primeiro domínio, os dados das manifestações emocionais dos cuidadores podem ser expressos pelos significados que os cuidadores atribuem ao nível de liberdade para deixar o exercício da função de cuidador, se assim desejassem em algum momento; as considerações próprias sobre o nível de dificuldade; a sensação de esgotamento e o nível do dilema interno suportado sobre o desempenho da responsabilidade com o idoso dependente, quesitos compreendidos no item "a" da avaliação de sobrecarga.

Através do item "b", o conhecimento sobre os desafios enfrentados por cuidadores de idosos ainda requer análise dos efeitos na vida particular do cuidador. Neste âmbito devem ser enfatizados os reflexos de sua atividade sobre seu quadro de saúde, para saber se os cuidados com idoso dependente o fizeram adoecer; o nível de exigência do trabalho destinado aos cuidados com idoso.

O item "b" de avaliação como prossegue em conhecer quais as conseqüências na esfera do controle de outras áreas da vida de seu interesse; sobre os planejamentos pessoais (mudanças ou conservação); no tempo destinado a si próprio; efeitos no nível de inconformidade com a situação de cuidador; repercussão na vontade por novos projetos pessoais; na sensação de aprisionamento; na rede de amizade e atividades sociais; nas programações recreativas; e sua percepção de isolamento. 
Segue-se que o levantamento de informações de sobrecarga se constitui igualmente da perspectiva das dificuldades econômicas enfrentadas pelo cuidador e suas projeções financeiras - item "c" -, ou seja, em se verificar quais as implicações dos cuidados prestados sobre as finanças no presente e nas expectativas futuras. Já quanto à avaliação das reações às exigências, referente aos quesitos do item “d”, aborda-se em qual medida o cuidador ficou zangado, sentindo-se ofendido diante do comportamento do familiar.

O modelo de avaliação de sobrecarga QIASCI apresenta o domínio do item “d”, em questão, complementando-se com dados acerca da frequência com que o cuidador se envergonhou do comportamento do seu familiar idoso, com dados sobre o nível de ocorrência de solicitações deste familiar em assuntos desnecessários. Adicionam-se a estes aspectos o quesito do senso de manipulação que supõe ser vítima e o índice de manifestação da inconformidade com a restrição de seus interesses privados (Martinset al., 2015).

Por sua vez, o item "e" se junta ao rol de informações de interesse do conhecimento de sobrecarga, a partir da autoavaliação de desempenho dos cuidados e vida pessoal. A análise desta perspectiva envolve o nível de controle e eficácia sobre os seguintes pontos: capacidade de realização de coisas pessoais que necessitam ser feitas; capacidade de continuação dos cuidados com o familiar sob os seus cuidados por um período mais longo de tempo; habilidades e conhecimentos técnicos sobre a patologia e cuidados que devem ser administrados; e o nível de reconhecimento dos familiares sobre sua dedicação.

Enquanto exploração dos elementos causadores de sobrecarga, a manutenção do enfoque da avaliação, que se dá na linha do propósito central dos desafios do cuidador, envolve a opinião do cuidador sobre o nível de apoio dos familiares (item "f”). Neste campo, são consideradas as manifestações de gratidão por parte do idoso sob os seus cuidados e de outros familiares.

O domínio que corresponde ao item "g" do questionário de avaliação de sobrecarga colhe o nível de satisfação do cuidador diante de reações positivas do idoso que reconhece pequenos gestos de atenção e carinho recebidos. Nesse sentido, se faz medição do alcance dos valores cultivados pelo cuidador, a respeito do seu trabalho, na dimensão da autoestima, ou seja:se avalia até que ponto o cuidador se sente uma pessoa especial, com mais valor.

Através da exposição dos componentes da estrutura de avaliação do QIASCI, a literatura consultada permitiu evidenciar associação dos elementos sociodemográfico com algum nível de sobrecarga. Todavia, verificou-se que outros elementos são tomados para efeito de investigação. Após a colheita desses dados, as pontuações são computadas, resultando na indicação do tipo de sobrecarga: se alta, média ou baixa.

Salienta-se que o instrumento descrito anteriormente se trata de um mecanismo entre vários empregados com a mesma finalidade. Existe outro indicador internacional posicométrico: o Zarit Burden Interviw ou Escala de Zarit. Em que pese seu desenvolvimento objetivado os cuidadores de paciente com demência, este outro sistema de medição de sobrecarga tem sido aplicado para cuidadores de pessoas com diversos diagnósticos. Contudo, a diversidade destes métodos de aferição tem dificultado a compreensão do panorama brasileiro acerca de sobrecarga de cuidadores de idosos, pois a literatura registra a existência de mais 14 tipos de métodos de avaliação (Barroso et al., 2017).

\section{Enfoque 2 - A sobrecarga nos cuidados com idosos com algum tipo de demência}

O tipo de cuidado com idosos com maior prevalência de sobrecarga se verifica quando o idoso está acometido de alguma demência (Leite et al., 2017; Soares et al., 2016). Nesse sentido, quanto à perspectiva de estudos em autopercepção, demonstrou-se que as dificuldades enfrentadas por cuidadores de idosos acometidos de demência variam caso a caso (Barbosa et al., 2019).

Assim, no contexto de cuidados aos idosos com demência, a avaliação do nível de sobrecarga dos cuidadores se torna um requisito para intervenção da enfermagem na forma de alternativas de atenuação, ou seja, a partir da identificação das causas da elevação de resistência à prestação dos cuidados, por causa do excesso de tarefas e jornada dos cuidados (Lino et al., 2016; Nunes et al., 2019).

\section{Enfoque 3 - Comparativo de sobrecarga entre cuidadores formais e informais, e o cuidador sob a orientação da equipe de Estratégia da Família}

A literatura examinada tem evidenciado que, entre os cuidadores informais, o desconhecimento da doença do idoso combinada à baixa instrução educacional do cuidador são fatores de sobrecarga. Em termos comparativos, colocando essa categoria ao lado dos cuidadores formais, que executam esse tipo de serviço seja de forma autônoma, seja por via de empresa com fins lucrativos ou por instituições filantrópicas, 
observa-se o seguinte: se por um lado há maior planejamento e preparo no domínio de algumas técnicas entre os cuidadores formais, por outro lado, a sobrecarga é ocasionada por jornada extensa de cuidados e maior quantidade de idosos sob a sua atenção. Deste modo, o sistema das instituições de longa permanência necessita de revisão de suas práticas, pois a sobrecarga alta, em cuidadores formais, interfere no êxito dos cuidados despendidos (Dinizet al., 2018; Barbosa et al., 2017b).

Com relação às Estratégias da Saúde da Família, a equipe integrante tem um papel de contribuir com atenuação da sobrecarga por meio de intervenções periódicas, dando orientação aos cuidadores informais familiares (Mendes et al., 2019; Muniz et al., 2016; Soares et al., 2016; Santos et al., 2019), porque os elementos causadores de sobrecarga são passíveis de superação quando identificados, e os cuidadores necessitam de orientações eventuais dos profissionais da saúde (Lopeset al., 2020; Nunes et al., 2019).

\section{Considerações finais}

Os 10 artigos analisados demonstraram, considerando a perspectiva do estado clínico do cuidador e seu perfil sociodemográfico, que os elementos de sobrecarga são: o acometimento de alguma patologia, uma variável que se apresenta como causa de elevação da sobrecarga; se o cuidador também é idoso; dificuldades financeiras; insuficiência ou inexistência de suporte familiar; e a percepção valorativa baixa do próprio papel de cuidador.

Por meio da descrição do método de avaliação QIASCI e das impressões das pesquisas selecionadas sobre elementos de sobrecarga, conclui-se que os cuidados se acentuam na medida em que os cuidadores são mulheres e cônjuges do idoso sob os seus cuidados. Já na perspectiva do tipo de patologia que o idoso seja portador, verificou-se que os cuidadores de idosos com demência são as circunstâncias de maior agravamento da sobrecarga.

\section{Referências}

Barbosa, T. B. A. G., Araújo, A. C. J., \& Lucena, E. F., 2017. Lei 13.466/2017: Preferência entre prioridades. In: Anais do Congresso Internacional de Direitos Difusos, Paraíba. Disponível em:<https://www.editorarealize.com.br/artigo/visualizar/30908>. Acesso em: 14 de abr. de 2020.

Barbosa, L. M., Noronha, K., Spyrides, M. H. C., \& Araújo, C. A. D. 2017b. Qualidade de vida relacionada à saúde dos cuidadores formais de idosos institucionalizados em Natal, Rio Grande do Norte. Revista Brasileira de Estudos da População, 34(2), 391-414.

Barbosa, M. E. M., Bertelli, E. V. M., Scolari, G. A. S., Bortolanza, M. C. Z., Higarashi, I. H., \& Careira, L. 2019. Vulnerabilidade clínica e funcional de idosos cuidadores de idosos com doença de Alzheimer. Revista Rene, 20, e40851.

Barroso, S. M., Santos, L. P., \& Francielle e Silva, L. 2017. Instrumentos de avaliação de sobrecarga em cuidadores informais: uma revisão integrativa. Avaliação Psicológica, 16(4), 498-504.

Botelho, L. L. R., Cunha, C. C. A., \& Macedo, M. 2011. O método da revisão integrativa nos estudos organizacionais. Gestão e Saúde, 5(11), 121-136.

Brandão, F. S. R., Costa, B. G. S., Cavalcanti, Z. R., Bezerra, M. R., Alencar, L. C., A., \& Leal, M. C. C. 2017. Sobrecarga dos cuidadores idosos assistidos por um serviço de atenção domiciliar. Revista de Enfermagem UFPE, 11(1), 272-279.

Brasil. Ministério da Saúde. 2010. Secretaria de Atenção à Saúde. Departamento de Ações Programáticas e Estratégicas. Atenção à saúde da pessoa idosa e envelhecimento. Brasília: Ministério da Saúde.

Brasil. Ministério da Saúde. 2008. Secretaria de Atenção à Saúde. Guia prático do cuidador. Brasília, Ministério da Saúde.

Diniz, M. A. A., Melo, B. R. S., Neri, K. H., Casemiro, F. G., Figueiredo, L. C., Gaioli, C. C. L. O., \& Gratão, A. C. M. 2018. Estudo comparativo entre cuidadores formais e informais de idosos. Ciência \& Saúde Coletiva, 23(11), 3789-3798.

Gonçalves, L. T. H., Leite, M. T., Hildebrandt, L. M., Bisogno, S. C., Biasuz, S., \& Falcade, B. L. 2013. Convívio e cuidado familiar na quarta idade: qualidade de vida de idosos e seus cuidadores. Revista Brasileira de Geriatria e Gerontologia, 16(2), 315-325.

Lana, L. D., \& Schneider, R. H. 2014. Síndrome de fragilidade no idoso: uma revisão narrativa. Revista Brasileira de Geriatria e Gerontologia,17(3), 673-80. 
Leite, B. S., Camacho, A. C. L. F., Joaguim, F. L., Gurgel, J. L., Lima, T. R., \& Queiroz, R. S. 2017. A vulnerabilidade dos cuidadores de idosos com demência: estudo descritivo transversal.Revista Brasileira de Enfermagem, 20(4), 682-688.

Lino, V. T. S., Rodrigues, N. C. P., Camacho, 1. A. B., O’Dwyer, G., Lima, I. S., Andrade, M. K. N., \& Atie, S.2016. Prevalência de sobrecarga e respectivos fatores associados em cuidadores de idosos dependentes, em uma região pobre do Rio de Janeiro, Brasil. Cadernos de Saúde Pública,32(6),e00060115.

Lopes, C. C., Oliveira, G. A., Stigger, F. S., \& Lemos, A. T. 2020. Associação entre a ocorrência de dor e sobrecarga em cuidadores principais e o nível de independência de idosos nas atividades de vida diária: estudo transversal.Cadernos Saúde Coletiva, 28(1), 98-106.

Loureiro, L. S. N., Fernades, M. G. M., Nóbrega, M. M. L., \& Rodrigues, R. A. P. 2014. Sobrecarga em cuidadores familiares de idosos: associação com características do idoso e demanda do cuidado. Revista Brasileira de Enfermagem, 67(2), 227-32.

Martins, T., Peixoto, M. J., Araújo, F., Rodrigues, M., \& Pires, F. 2015. Desenvolvimento de uma versão reduzida do questionário de avaliação de sobrecarga do cuidador informal. Revista da Escola de Enfermagem da USP, 49(2), 236-244.

Mendes, P. N., Figueiredo, M. L. F., Santos, A. M. R., Fernandes, M. A., \& Fonseca, R. S. B. 2019.Sobrecargas física, emocional e social dos cuidadores informais de idosos. Acta Paulista de Enfermagem, 32(1), 87-94.

Monteiro, E. A., Mazin, S. C., \&Dantas, R. A. S. 2015. Questionário de avaliação de sobrecarga do cuidador informal: validação para o Brasil. Revista Brasileira de Enfermagem, 68(3), 421-428.

Muniz, E. A., Freitas, C. A. S. L., Oliveira, E. N., \& Lacerda, M. R. 2016. Grau de sobrecarga dos cuidadores de idosos atendidos em domicílio pela Estratégia Saúde da Família. Saúde em Debate, 40(110), 172-182.

Nunes, S. F. L., Alvarez, A. M., Costa, M. F. B. N. A., \& Valcarenghi, R. V. 2019. Fatores determinantes na transição situacional de familiares cuidadores de idosos com doença de parkison. Texto \& Contexto Enfermagem, 28, e20170438.

OPAS. Organização Pan-Americana de Saúde. 2018. Folha informativa - Envelhecimento e saúde. Brasília, 2018. Disponível em:<https://www.paho.org/bra/index.php?option=com_content\&view=article\&id=5661:folhainformativa-envelhecimento-e-saude\&Itemid=820>. Acesso em: 03 de mar. 2020.

Santos, W. P., Freitas, F. B. D., Sousa, V. A. G., Oliveira, A. M. D., Santos, J. M. M. P., \& Gouveia, B. L. A. 2019. Sobrecarga de cuidadores idosos que cuidam de idosos dependentes. Revista Cuidarte, 10(2), e607.

Soares, C. B., Hoga, L. A. K., Peduzzi, M., Sangaleti, C., Yonekura, T., \& Silva, D. R. A. D. 2014. Revisão integrativa: conceitos e métodos utilizados na enfermagem. Revista da Escola de Enfermagem da USP, 48(2), 335-345.

Soares, R. T., Ferreira, C. G., Dias, V. N., Lemos, N. F. D., \& Gazzola, J. M. 2016. Cuidando do paciente com Síndrome Demencial: a sobrecarga do cuidador principal.RevistaKairós, 19(2), 309-325.

Yamashita, C. H., Gaspar, J. C., Amendola, F., Alvarenga, M. R. M., \& Oliveira, M. A. C.2014. Rede social de cuidadores familiares de pacientes com incapacidades e dependência. Revista da Escola de Enfermagem da USP, $48,097-103$.

\section{Minicurrículo}

Iasmim Sofia Terceiro Nunes. Enfermeira pela Universidade Ceuma (2021). Pós-graduanda em Saúde da família e UTI neonatal e pediátrica pela Faculdade Laboro, atuando principalmente nos seguintes temas: enfermagem, saúde coletiva, saúde do idoso, saúde do RN e cuidado a saúde da pessoa idosa.

Gilmárcia Pinheiro Pereira. Enfermeira pela Universidade Ceuma (2005), especialização em Saúde da Família pela Universidade Castelo Branco. Atua como preceptora de enfermagem da UNICEUMA em diversas unidades de saúde de São Luís, Maranhão. Mestranda em Meio Ambiente pela Universidade Ceuma. 
Como citar: Nunes, I.S.T., \& Pereira, G. P. 2021. Desafios enfrentados por cuidadores de idosos: uma revisão integrativa. Pubsaúde, 7, a254. DOI: https://dx.doi.org/10.31533/pubsaude7.a254

Recebido: 16 set. 2021.

Revisado e aceito: 26 set. 2021.

Conflito de interesse: os autores declaram, em relação aos produtos e companhias descritos nesse artigo, não ter interesses associativos, comerciais, de propriedade ou financeiros que representem conflito de interesse.

Licenciamento: Este artigo é publicado na modalidade Acesso Aberto sob a licença Creative Commons Atribuição 4.0 (CC-BY 4.0). 